\title{
New scaling parameter for turbulent boundary layer with large roughness
}

\author{
C. S. Subramanian \& M. Lebrun \\ Department of Mechanical and Aerospace Engineering, \\ Florida Institute of Technology, Melbourne, Florida
}

\begin{abstract}
Nikuradse uses the equivalent sand-grain roughness to characterize the effect of roughness. While this approach works when the roughness is contained in the inner layer, it does not apply in recent studies with a larger roughness. Various techniques have been applied in the past to scale the mean velocity and the Reynolds stress profiles for a zero pressure gradient boundary layer, the classical scaling using the friction velocity $\mathrm{u}^{*}$ to normalize the velocity profiles. However none of these techniques holds universally.

This study attempts to improve the understanding that we have of the way roughness affects the inner layer behaviour and aims to find an alternative scaling parameter for cases where roughness is large compared to the inner layer. Measured mean and turbulent velocity profiles on a large regular roughness show a non-zero wall normal pressure is caused which contributes to the velocity deficit in the near wall rough boundary layer velocity profile. The normal turbulent stresses are also increased. Hence a pressure gradient velocity rather than the friction velocity is defined to capture the pressure effects induced by roughness. The power law seems to give a better representation of the velocity profiles than the log law in this case.

Keywords: large roughness, boundary layer, friction velocity, turbulent velocity, pressure gradient velocity, log law, power law.
\end{abstract}

\section{Introduction}

Some of the most recent studies of the effects of surface roughness on boundary layer structure were performed by Perry et al. [2], Bandyopadhyay and Watson [3], Barenblatt [4], Acharya et al. [5] and Keirsbulk et al. [6]. Roughness may be 
classified in various ways depending on the geometry of the roughness elements. It may be deterministic (regular) or stochastic (random). Most of the research has been done on "regular" roughness patterns such as sand grain roughness.

Nikuradse [1] and Schlichting [7] were the first ones to introduce the concept of equivalent sand grain roughness size, ks, to characterize and quantify roughness effects on the boundary layers. But in many cases, a single parameter is not enough to characterize the hydrodynamics of a surface. The logarithmic velocity profile relationship is stated as:

$$
\frac{u}{u^{*}}=\frac{1}{\kappa} \ln \left(\frac{y \cdot u^{*}}{v}\right)+C
$$

where, $u^{*}$ is the friction velocity, $\kappa$ is the Karman constant, $C$ is the additive constant.

The classical framework established by Nikuradse [1] predicts that the effect of roughness on the mean-velocity distribution is confined to a thin wall layer. Many researchers have verified through experiments the existence of the log-law region of the boundary layer for flows with a range of pressure gradients (Klebanoff and Diehl [8]; Clauser [9]; and many others). However values of $C$ and $\kappa$ are still debated.

Durbin and Belcher [12] defined a viscous pressure-gradient velocity which is derived in Subramanian's research [13] where he shows that if you define the characteristic velocity as $u_{p}$ given by

$$
u_{p}^{3}=\frac{v}{\rho} \cdot \frac{d P_{s}}{d x}
$$

where $P_{s}$ is the surface mean pressure, independent of y but function of $\mathrm{x}$, an equation can be derived with only one characteristic velocity $u_{p}$ and one characteristic length scale $v / u_{p}$, and its boundary conditions are homogeneous (both $u$ and the shear stress are zero at $\mathrm{y}=0$ ) which would yield a solution similar to the law-of-the-wall as (1).

$u_{p}$ seems to be a better scale in situations where the friction velocity, $u^{*}$, is ill defined or tends to zero and the local pressure gradient is the influencing parameter.

The purpose of this research was to gain some fundamental and practical knowledge of these flows and to find an alternative scaling parameter for flows where the roughness is very strong and where the "log law" does not apply anymore.

\section{Experimental set up and method}

All the experiments were conducted in the boundary layer wind tunnel located at the FloridaTech laboratories.

The wind tunnel test section is $1.7 \mathrm{~m}$ in length, $0.54 \mathrm{~m}$ in width and $0.54 \mathrm{~m}$ in height. Three test plates $(0.34 \mathrm{~m} \times 0.27 \mathrm{~m})$ were designed to go on the boundary layer plate as an insert. The first one follows the smooth pattern of the plate, the 
second one has the two-dimensional roughness, and the third one has the threedimensional rough elements. Figure 1 shows where the roughness is located on the boundary layer plate. The type of roughness used is k type, where the ratio of the gap width over the height of the roughness elements is greater than 1 . The gap width is $7 \mathrm{~mm}$, the height is $6 \mathrm{~mm}$. Each roughness element width is $3 \mathrm{~mm}$. The plate containing the 3D roughness has pressure taps in both $\mathrm{x}$ (longitudinal) and the $\mathrm{z}$ (transverse) directions to measure the pressure at all the experimental points. A trip wire was attached at $1 \mathrm{~cm}$ from the leading edge in order to obtain a fully turbulent flow over the plate.

The boundary layer test section is fitted with a boundary layer flat plate. The leading edge of the test plate was shaped to mimic the forward position of an airfoil. In order to measure the boundary layer pressure gradient, 3 surface static pressure taps were placed on the plate; more were placed on the inserts. Table 1 gives the location of the pressure taps, including on the insert.

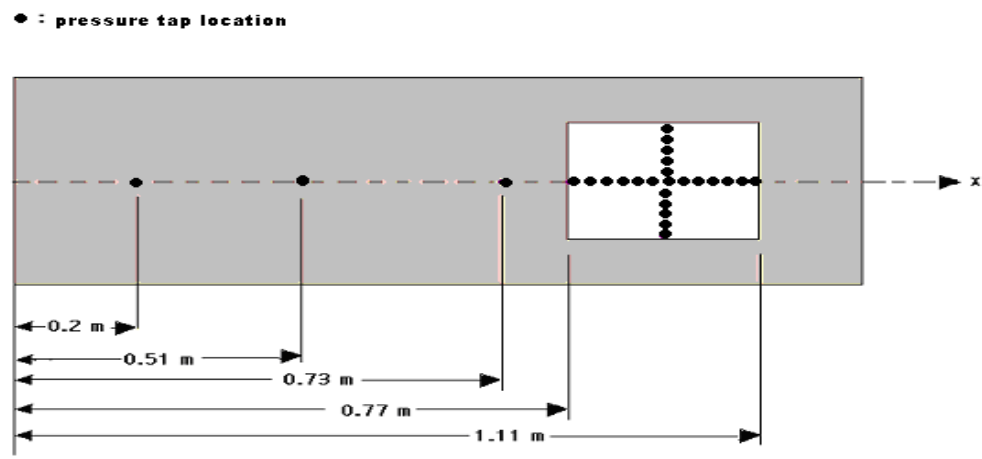

Figure 1: $\quad$ Location of pressure taps on the boundary layer plate.

Table 1: $\quad$ Location of the pressure taps on the 3 inserts in $\mathrm{cm}$.

\begin{tabular}{|c|c|c|c|c|c|c|c|c|c|c|}
\hline Smooth & 20 & 51 & 73 & 81.5 & 86 & 90.5 & 95 & 99.5 & 104 & \\
\hline 2D & 77.5 & 79 & 80.5 & 83 & 85.5 & 87 & 89.5 & 91 & 92.5 & 95 \\
\cline { 2 - 12 } Rough & 96.5 & 98 & 100.5 & 102 & 103.5 & 106 & 107.5 & 109 & 110.5 & \\
\hline \multirow{3}{*}{$\begin{array}{c}\text { 3D } \\
\text { Rough }\end{array}$} & 77.5 & 79 & 80.5 & 82 & 84 & 85.5 & 87 & 89 & 90.5 & 92 \\
\cline { 2 - 12 } & 94 & 95.5 & 97 & 99 & 100.5 & 102 & 104 & 105.5 & 107 & \\
\cline { 2 - 11 } & -14 & -12.5 & -10.5 & -9 & -7.5 & -5.5 & -4 & -2 & 0 & \\
\cline { 2 - 10 } & 1.5 & 3 & 5 & 6.5 & 8 & 10 & 11.5 & 13 & 14.5 & \\
\hline
\end{tabular}

Three probes were used to measure the boundary layer profile. The first probe was a boundary layer pitot tube which measured total pressure, in conjunction with the surface static pressure taps located on the plate. The second probe was a hotwire probe Model TSI 1218-10 serial B460 with a 5-micron diameter single fibre-film probe used in conjunction with a Dantec 56C17 constant temperature anemometer. It is made of platinum film and has a nominal resistance of $5.30 \mathrm{ohms}$. The third probe is an $\mathrm{x}$-wire (Model 55R53) mounted on a Dantec long probe support (Model 55H25) and used in conjunction with the 
Dantec 56C17 constant temperature anemometer. This probe has a nominal resistance of $4.99 \mathrm{ohms}$ and $4.74 \mathrm{ohms}$. Each probe was attached to a vertical traverse to take data at different height locations. The initial location from the surface was determined approximately by eyeballing which introduces offset errors. The first position of the pitot probe was taken to be 0.63 times the diameter thickness of the probe to account for the wall proximity error on the velocity. The probes were traversed from 0 to $20 \mathrm{~mm}$ in $1 \mathrm{~mm}$ intervals then from 20 to $50 \mathrm{~mm}$ in $2 \mathrm{~mm}$ intervals. The traverse's stepper motor resolution is $0.127 \mathrm{~mm}$. The probe traversing and the data acquisition were automated. The data acquisition system used for this experiment consisted of National Instruments (NI) PCI 6024E 100ks/s multi-function card, Lab View 6.0 software, and a personal computer. Data acquisition from the pressure transducer was done at 1,000 samples/second with a total of 10,000 samples, which were used for velocity statistics. Data acquisition from the anemometer was set at 10,000 samples/second with a total of 20,000 samples, which were used for velocity statistics.

\section{Results and discussion}

Pitot tube, pitot static tube, Preston tube, single wire anemometry, cross-wire anemometry and visualization techniques were performed to obtain measurements. The data uncertainty for the probes are $6 \%$ for the pitot static tube, $14 \%$ for the Preston probe, $2 \%$ for the hotwire and $3 \%$ for the $\mathrm{x}$-wire. At all time of the experiment ambient conditions were noted. The average temperature among all the experiments was $24.0^{\circ} \mathrm{C}$ with an average relative humidity of $71 \%$ and an average atmospheric pressure of $1015.0 \mathrm{hPa}$. The following air properties are derived from the above listed ambient conditions. The average air density was $1.189 \mathrm{~kg} / \mathrm{m}^{3}$, the average absolute viscosity was $1.839-05 \mathrm{Nm}^{2} / \mathrm{s}$ and the average kinematic viscosity was $1.547 \mathrm{E}-05 \mathrm{~m}^{2} / \mathrm{s}$. The boundary layer thickness, $\delta 99$, and the skin friction coefficient, $\mathrm{C}_{\mathrm{f}}$, estimated by the Clauser method give values that are consistent with the empirical correlation results found in many textbooks for a nominal smooth-wall turbulent boundary.

For estimating the $u_{p}$ values, the longitudinal surface pressure gradients are measured with respect to the immediate upstream tap. The surface pressure distribution was checked along the plate at 15 different points to determine the differences caused by the roughness. Figure 2 shows the plot of the surface pressure distribution along the centerline on the $2 \mathrm{D}$ and on the $3 \mathrm{D}$ roughness. The dynamic pressure is plotted and the reference pressure used is $\mathrm{P}_{\propto}$. The accuracy of the pressure measurements is $\pm 5 \mathrm{~Pa}$. The magnitude of the surface pressure is small but it is clearly non-zero and increases with respect to the free stream on the local roughness. Figure 2 shows that the pressure increases rapidly at the very first point on the roughness then decreases on the rough surface. The pressure stabilizes around the smooth plate value far downstream. The estimated pressure velocities, although relatively small, are considered a better indicator of the local pressure change. Therefore the surface pressures should be measured at 
close intervals and with a sensitive transducer to get a good estimation of the pressure velocity for surfaces where variations due to roughness are large.

Extensive flow measurements were taken first on the smooth plate then on the $2 \mathrm{D}$ rough plate to compare the two. Measurements were performed with the pitot tube, the hotwire and the $\mathrm{x}$-wire measuring $\mathrm{u}$ and $\mathrm{v}$ components at 10 stream wise locations on the rough plate. The $u$ velocity deficit in the inner layer increases with the downstream distance, which suggests that the inner layer flow adjusts to the smooth wall condition only further downstream. The profiles are in agreement with previous finding that the overlap region is the most affected by the roughness. The longitudinal component of turbulent direct stress increases as much as twice the value of the smooth wall near the wall by the roughness. The stress level increases as we go downstream, showing that it extends in to the outer region. This might be due to enhanced diffusion. All profiles asymptote to the same value in the outer layer. The same observations can be made for the normal component of the turbulent direct stress. The normal component of the turbulent direct stress is mostly responsible for inducing the normal pressure gradients along the wall. There is more increase of this stress due to roughness than of the longitudinal component of direct stress. The shear stress profiles in Figure 3 show that the stress level is higher near the wall due to roughness. The constant shear stress region that is usually present in the smooth wall profiles is practically non-existent. Therefore the wall similarity law may not be used in this case. The peak stress occurs closer to the wall than for a smooth wall, therefore abrupt velocity gradients are expected at the wall when the roughness is strong.

The results obtained on the $3 \mathrm{D}$ roughness are very similar. It seems that the flow is even less turbulent than with the 2D roughness. Measurements were taken with the pitot tube and the hotwire at 8 stream wise locations, along the centre line, alternatively on and within the roughness, at 10 and $15 \mathrm{~m} / \mathrm{s}$ free stream speeds. Velocity profiles can be seen on Figure 4 and we can conclude that along the roughness, the $\mathrm{u}$ velocity near the wall is dramatically reduced compared to smooth wall. For 3D roughness, as well as for $2 \mathrm{D}$ rough elements, the coefficient of friction cannot be found using the Clauser chart for the log law does not exist anymore.

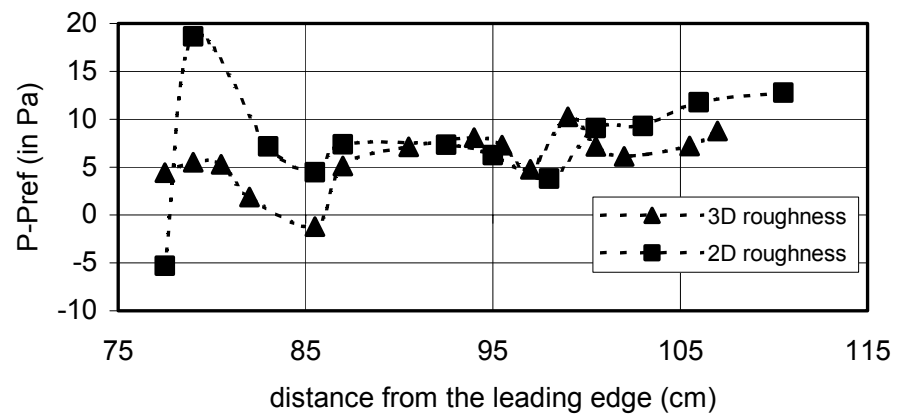

Figure 2: $\quad$ Variation of surface pressure with roughness at $10 \mathrm{~m} / \mathrm{s}$. 


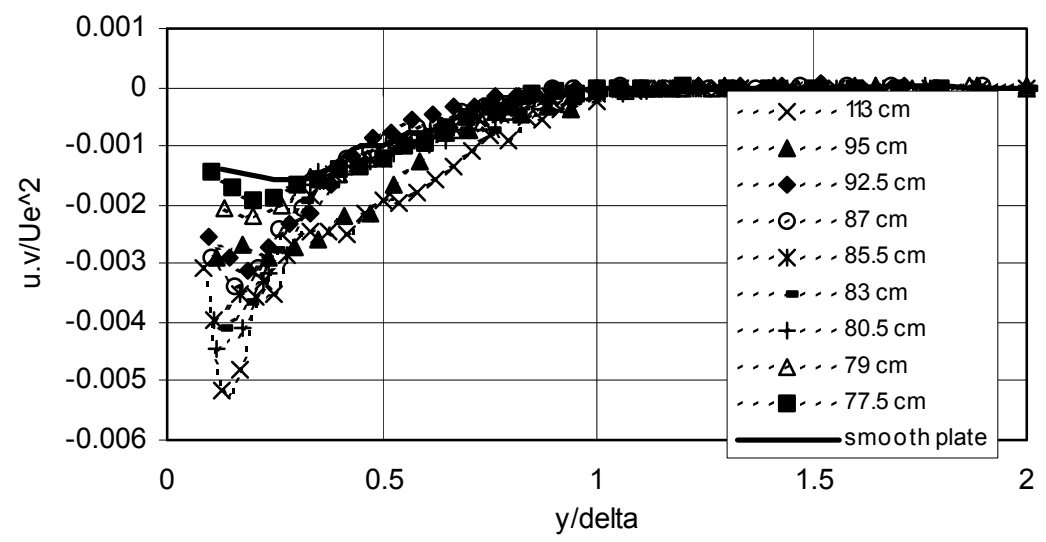

Figure 3: Turbulent shear stress profiles along the centreline.

For the 3D roughness, the velocity profiles in the $\mathrm{z}$ (transverse) direction are shown in Figure 5. On the rough plate, the flow does not follow the $1 / 7^{\text {th }}$ power law. There is no pattern as we go further left or further right from the centreline. The mean profile is laminar like unlike what we saw on the $2 \mathrm{D}$ insert. It seems that the 3D roughness, since it has less blockage than the 2D roughness (the roughness elements are now cubes that allow more air to flow between them) turns the mean velocity profile into a laminar profile but we cannot consider the flow as laminar because it still has a high turbulent intensity.

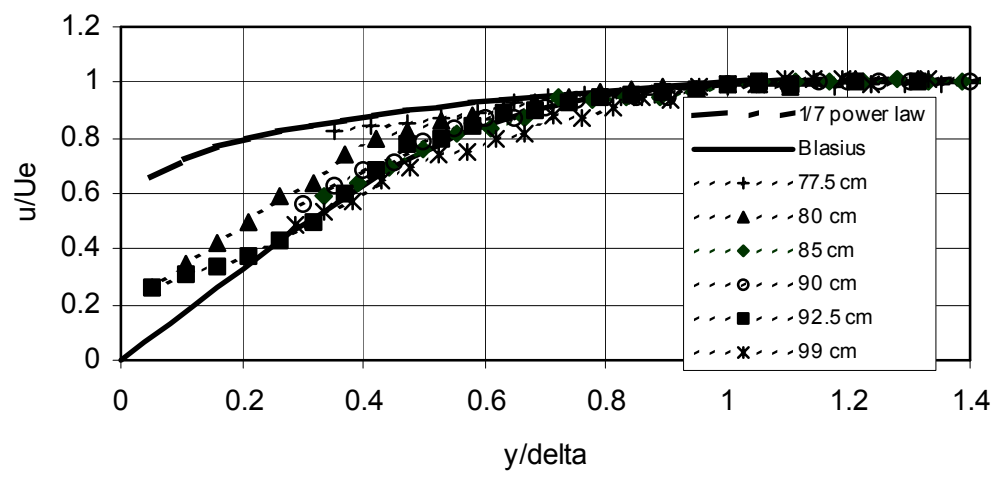

Figure 4: u-velocity profile development on 3D roughness with hotwire.

We are looking for a better scaling parameter for cases where the roughness is large. The parameter $u_{p}$ defined earlier seems to be a better scaling parameter than the usual skin friction velocity $u^{*} . u_{p}$ is calculated using Eq. (2). The pressure transducer gives us series of surface pressure measurement, from that 
the static pressure is found at each experimental point and $d P_{s} / d x$ can be calculated. From there we can deduce $u_{p}$ at each experimental point.

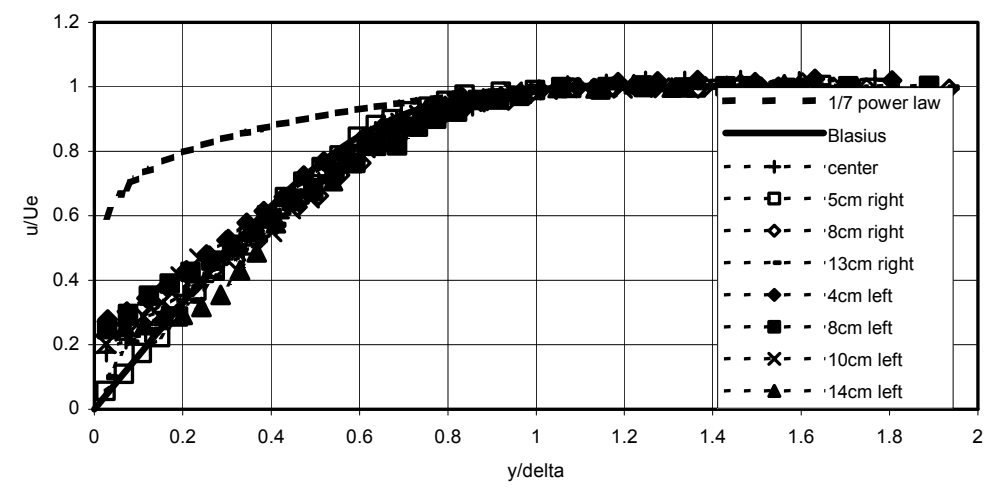

Figure 5: Transversal velocity across the $3 \mathrm{D}$ plate at $\mathrm{x}=92 \mathrm{~cm}$ and $15 \mathrm{~m} / \mathrm{s}$.

Figure 6 shows the distribution of $u_{p}$ on the $2 \mathrm{D}$ rough plate at $10 \mathrm{~m} / \mathrm{s}$. There does not seem to be a pattern but on the other hand, we cannot conclude anything for the points contained within the limits of uncertainty. The uncertainty on the pressure is $\pm 5 \mathrm{~Pa}$ with gives us an uncertainty on $\mathrm{u}_{\mathrm{p}}$ of $\pm 0.13 \mathrm{~m} / \mathrm{s}$. Figure 7 shows a semi log plot of the longitudinal mean velocity profiles plotted using the new scaling parameter $u_{p}$ both on the smooth and on the rough surface. A linear log-law region is noticeable but a more accurate estimate of the $u_{p}$ might be needed. Stratford's zero-wall shear stress velocity profile gives a log-law distribution with a similar scaling with a slope of 5 and an intercept of 8 . In our case, the wall shear stress is not zero and therefore we do not have a first order equation: a second order velocity correction term is required.

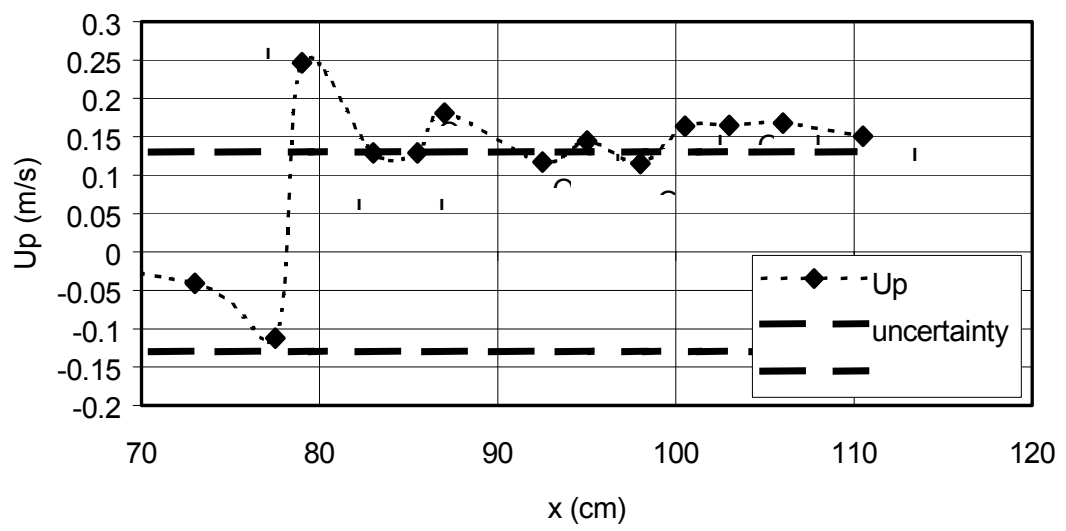

Figure 6: $u_{p}$ distribution on the $2 D$ rough plate at $10 \mathrm{~m} / \mathrm{s}$. 


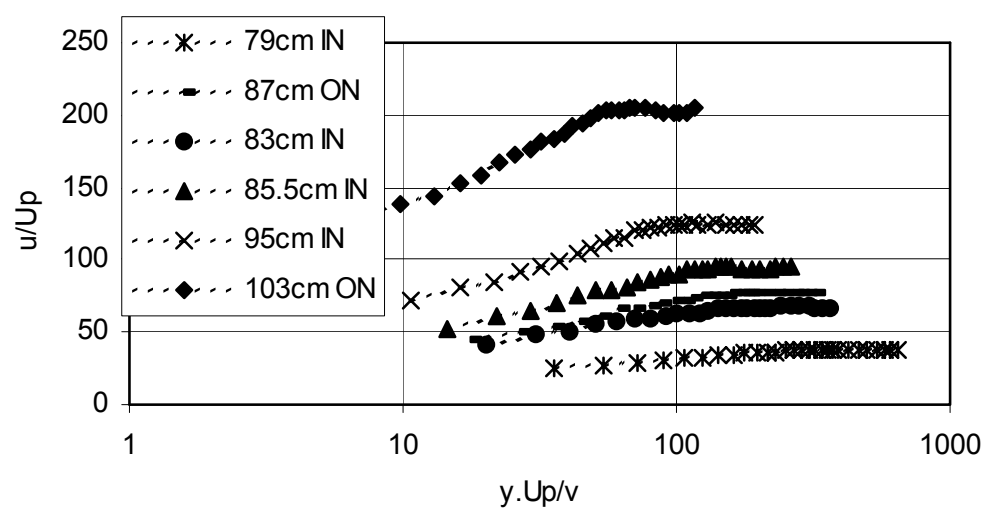

Figure 7: Longitudinal mean velocity profiles based on $u_{p}$ scaling, $z=0$.

If we plot the shear stress profile using the $u_{p}$ and $v / u_{p}$ normalization, the negative peak magnitude of shear stress increases and moves away from the surface with the increase of streamline distance. Downstream of the roughness, some constant shear stress region is seen. We can see that the $\mathrm{u}, \mathrm{v}$ and $\mathrm{w}$ fluctuations are greater close to the wall (i.e. the inner layer) compared to the smooth wall data. Away from the wall, the fluctuations seem to decrease as the distance from the leading edge increases therefore the outer layer of the turbulent boundary layer are affected by the roughness.

The span wise size of the roughness elements plays an important role in determining whether the outer layer is going to be altered by the roughness or not. The local roughness can be expressed in different ways, as suggested by Subramanian et al. [13] to define the roughness using $\mathrm{Ra}$, the average roughness height over a square $\mathrm{mm}$ area, Rt, the difference between the largest positive deviation and the largest negative deviation from the mean line in a $1 \mathrm{~mm}$ square area and $\mathrm{Rq}$ the root mean square roughness.

We find: $\mathrm{Rt}=6 \mathrm{~mm}$ and $\mathrm{Rq}=4.24 \mathrm{~mm}$ for the $2 \mathrm{D}$ and the $3 \mathrm{D}$ roughness. $\mathrm{Ra}$ is $6 \mathrm{~mm}$ on the 2D roughness and $3 \mathrm{~mm}$ on the 3D plate. The ratio Ra/Rt appears to be a good indicator of the roughness, for the $2 \mathrm{D}$ roughness, $\mathrm{Ra} / \mathrm{Rt}$ is 1 meaning that the local peaks and valleys are of the same size. For the 3D roughness, $\mathrm{Ra} / \mathrm{Rt}$ is 0.5 showing that the roughness occupies only half the area that it did in the $2 \mathrm{D}$ case. Recent research has been done on scaling the power law velocity profile, which is a good alternative to the log law in cases like ours with large roughness. Afzal [14] proposes the relation and Barenblatt et al. [15] the correlations of power law constants:

$$
\begin{gathered}
\frac{u}{U^{*}}=C\left(\frac{y \cdot U^{*}}{v}\right)^{\alpha} \\
\alpha=\frac{3}{2 \cdot \ln R_{e}} \text { and } C=\frac{1}{\sqrt{3}} \ln R_{e}+\frac{5}{2}
\end{gathered}
$$

If we apply Eq. (3) using Barenblatt's coefficients (Eq. (4)) and using $u_{p}$ instead of $\mathrm{u}^{*}$, we get a perfect correlation on the $2 \mathrm{D}$ roughness as can be seen on 
Figure 8. Same thing for the 3D roughness. We obtain 2 nearly identical linear fits. This results proves that $u_{p}$ is a more adequate velocity scaling parameter than $\mathrm{u}^{*}$ which cannot be found easily on the rough surfaces.

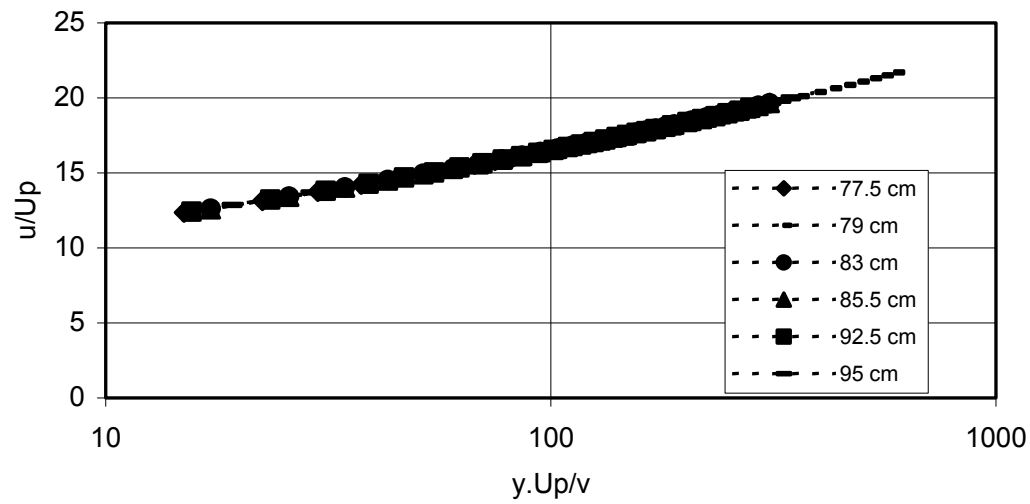

Figure 8: Longitudinal mean velocity profiles based on the scaling law and the scaling parameter $\mathrm{u}_{\mathrm{p}}$ on the $2 \mathrm{D}$ roughness.

\section{Conclusions}

Surface roughness is a defining feature of many of the high Reynolds-numbers flows found in engineering. The higher the Reynolds number, the more significant will the effects of roughness be. Unfortunately, the impact of surface roughness is not entirely understood. The turbulent boundary layer over a rough surface contains a roughness sublayer within which the flow is directly influenced by the local roughness elements and is therefore not spatially homogeneous. The height of this sublayer presumably depends upon the height of the roughness elements as well as their shape and density distribution in the lateral directions.

The rough-wall boundary layers can be categorized according to whether or not the surface roughness affects the outer layer. In this region, the inner log-law may be altered or destroyed, the existence of the log-law cannot be assumed anymore. All the scaling laws used so far are based only on its effect on the viscous drag even though several recent studies suggest that the turbulence structural changes caused by roughness are very profound in the inner layer.

The specific conclusions of this research are that the large regular roughness decreases the mean longitudinal velocity in the near wall layers and affects the overlap log-law layer. The mean velocity profiles follow the $1 / 7^{\text {th }}$ power law on the smooth plate insert, the $1 / 4^{\text {th }}$ power law on the $2 \mathrm{D}$ rough insert and the Blasius-like profile on the $3 \mathrm{D}$ rough insert. Large roughness increases all the turbulence quantities in the inner layer; the shear stresses are increased by a factor of 2 times the smooth wall value. There seems to be an effect of roughness not only on the inner layer but also on the outer layer structure of the boundary layer. The roughness causes a normal pressure gradient, invalidating the thin boundary layer assumption. The near wall pressure is higher than the free stream 
pressure. Inner layer recovery of the mean flow is slow downstream of the roughness. This research shows that the log law is not valid in cases were the roughness is large and it is necessary to find a scaling parameter that will work in those cases. A new scaling parameters was found and applied to our experimental data but, we still need to do more research to find a scaling parameter that can be applied to any boundary layer analysis.

The validity of the experiment and sources of error were confirmed. Among these is the inability to measure velocities near the surface, at less than $0.25 \mathrm{~mm}$ and without introducing flow obstructions.

\section{References}

[1] Nikuradse, J.: Laws of flow in rough pipes, NACA TM 1292, National Advisory Committee on Aeronautics, 1933.

[2] Perry, A.E., Lim, K. L. and Henbest, S. M.: An experimental study of the turbulence structure in smooth and rough wall boundary layers, J. Fluid Mech. 177, pp. 437-466, 1987.

[3] Bandyopadhyay, Promode R. and Watson, Ralph D.: Structure of roughwall turbulent boundary layers, Phys.Fluids 31, pp.1877-1883, 1988.

[4] Barenblatt G.I.: Turbulent Boundary Layers at a very large Reynolds number, Lawrence Berkeley National Laboratory, California, 2003.

[5] Acharya M., Bornstein J. and Escudier M.P.: Turbulent Boundary Layers on Rough Surfaces, Exp. Fluids, 4, pp. 33-47, 1986.

[6] Keirsbulck L., Labraga L., Mazouz A. and Tournier C.: Surface Roughness Effects on Turbulent Boundary Layer Structures, ASME J. Fluids Eng. 124, pp. 127-135, 2003.

[7] Schlichting, H.: Experimental investigation of the problem of surface roughness, NACA TM 832, National Advisory Committee on Aeronautics, 1936.

[8] Kebanoff, P.S. and Diehl, Z.W., Natl. Advisory Comm. Aeronaut. Tech. Notes. No. 2475, 1951

[9] Clauser, F. H., "The turbulent boundary layer," Adv. Appl. Mech. 4, pp. 1--51, 1956

[10] White, Frank M.: Viscous Fluid Flow, Second edition, McGraw-Hill, N.Y., 1991.

[11] Townsend A.A.: The structure of turbulent shear flow, Second edition, Cambridge University Press, Cambridge, 1976.

[12] Durbin P.A and Belcher S.E.: Scaling of adverse-pressure-gradient turbulent boundary layers, J. Fluid Mech., 238, pp. 699-722, 1992.

[13] Subramanian, Chelakara S., King, Paul I., Reeder, Mark F., Ou, Shichuan, Rivir, Richard B.: Effects of Strong Irregular Roughness on the Turbulent Boundary Layer, Flow, Turbulence \& Comb, 74(2-4), pp.349-368, 2004.

[14] Afzal, Noor: Scaling of Power Law Velocity Profile in Wall-Bounded Turbulent Shear Flows, 43 ${ }^{\text {rd }}$ Aerospace Science Meeting, Reno, NV, 2005.

[15] Barenblatt, G.I., Chorin, A.J., Prostokishin, V.M.: A model of a turbulent boundary layer with a nonzero pressure gradient, Proc. US NAS, 99(9), pp. 5772-5776, April 2002. 